Research Article

\title{
Oxytocin and arginine vasopressin systems in the domestication process
}

\author{
Bibiana S. O. Fam ${ }^{1}$, Pamela Paré ${ }^{1}$, Aline B. Felkl ${ }^{1}$, Pedro Vargas-Pinilla ${ }^{1}$, Vanessa R. Paixão-Côrtes ${ }^{1}$, \\ Lucas Henriques Viscardi ${ }^{1}$ and Maria Cátira Bortolini ${ }^{1}$ \\ ${ }^{1}$ Departamento de Genética, Universidade Federal do Rio Grande do Sul, Porto Alegre, RS, Brazil.
}

\begin{abstract}
Domestication is of unquestionable importance to the technological revolution that has given rise to modern human societies. In this study, we analyzed the DNA and protein sequences of six genes of the oxytocin and arginine vasopressin systems (OXT-OXTR; AVP-AVPR1a, AVPR1b and AVPR2) in 40 placental mammals. These systems play an important role in the control of physiology and behavior. According to our analyses, neutrality does not explain the pattern of molecular evolution found in some of these genes. We observed specific sites under positive selection in AVPR1b $(\omega=1.429, p=0.001)$ and AVPR2 $(\omega=1.49, p=0.001)$, suggesting that they could be involved in behavior and physiological changes, including those related to the domestication process. Furthermore, AVPR1a, which plays a role in social behavior, is under relaxed selective constraint in domesticated species. These results provide new insights into the nature of the domestication process and its impact on the OXT-AVP system.
\end{abstract}

Keywords: Oxytocin and receptors, vasopressin and receptors, animal domestication, molecular evolution, positive selection.

Received: May 4, 2017; Accepted: October 1, 2017.

\section{Introduction}

The phenomenon of domestication did not go unnoticed by Charles Darwin. With The Variation of Animals and Plants under Domestication, published in January 1868 , he devoted a whole book to the mechanisms underlying this intriguing process (Darwin, 1868). It is now well known that the complex process of animal domestication involves systematic selective pressures imposed by humans according to their needs and wishes. In a classic experiment modeling domestication, Russian researchers subjected silver foxes to rigorous artificial selection for tameness and correlated traits over more than 50 years. Although part of the lineages retained their ancestral traits, foxes selected for tamability showed a loss of wild-type behavior within relatively few generations, acquiring several classical morphological attributes of domesticated animals such as white spotting, floppy ears, and curly tails (Trut et al., 2009). The selection of traits, such as tameness and the reduction of innate stress, aggression, fear and anxiety, allowed domesticated animals to coexist and coevolve with humans within their constructed niches. This complex process arguably contributed to the rapid spread of our species across the globe, as well as facilitating the emergence of our civilization (Künzl and Sachser, 1999; Wiener and Wilkinson, 2011; Wright, 2015).

Send correspondence to: Maria Cátira Bortolini. Departamento de Genética, Universidade Federal do Rio Grande do Sul, Caixa Postal 15053, 91501-970 Porto Alegre, RS, Brazil. E-mail: maria.bortolini@ufrgs.br.
In recent years, a picture of the genetic basis for domestication has started to emerge (Schubert et al., 2014, Park et al., 2015; Marsden et al., 2016). Genes of the immune system, neuronal development and behavior were shown to have been co-opted as part of the domestication process (Albert et al., 2012; Wright, 2015). For instance, gray wolves (Canis lupus lupus) and dogs (Canis lupus familiaris) are highly differentiated in their expression of serotonin receptor genes, consistent with behavioral changes as part of domestication (Li et al., 2013). Importantly, domestication can lead to both the rapid fixation of alleles associated with phenotypes of interest and a relaxation of selective constraints previously imposed by natural selection, as has been described for dogs, horses (Equus caballus) and cows (Bos taurus) (Zeder, 2012).

In placental mammals, the paralogous nonapeptides oxytocin (OXT: Cys-Tyr-Ile-Gln-Asn-Cys-Pro-Leu-Gly) and arginine vasopressin (AVP: Cys-Tyr-Phe-Gln-AsnCys-Pro-Arg-Gly) play an important role in physiological and behavioral functions, such as water homeostasis, vasoconstriction, lactation, uterine contractions, parental care, control of aggression, anxiety and stress (Gimpl and Fahrenholz, 2001; Cagliani et al., 2009; Young and FlanaganCato, 2012; McCall and Singer, 2012). In addition to the respective nonapeptides, the genes $O X T$ and $A V P$ encode the carrier protein neurophysin (NP) and a signal peptide (SP). While the nonapeptides OXT and AVP are relatively well conserved in placental mammals, neurophysin and the signal peptide are more variable. The patterns of variation found in the portion of the $O X T$ and $A V P$ genes encoding 
NP and SP are therefore central to our question of linking genetic variation to domestication. Because both NP and SP play an important role in the export of the nonapeptides to their specific site of action, variation in these proteins might provide a mechanism by which OXT and AVPmediated responses differ, even in the face of nonapeptide sequence conservation.

OXT and AVP are produced in high quantities in the brain, but their systemic and/or tissue/organ specificity depends on an adequate interaction with NP and SP and their respective paralog G protein-coupled receptors (GPCRs), OXTR, AVPR1a, AVPR1b and AVPR2 (Kimura et al., 1992; Yamashita and Kitano, 2013). Nonetheless, it is known that some level of crosstalk can occur in the interaction of these ligands and their receptors (Zingg and Laporte, 2003; Slusarz et al., 2013). Like other GPCRs, OXTR, AVPR2, AVPR1a and AVPR1b all contain seven transmembrane domains (named TM1-TM7) as well as four extracellular (N-terminal tail-ECL3) and four intracellular (ICL1-C-terminal tail) domains. The N-terminal tail is crucial for the detection of molecules outside the cell, while the $\mathrm{C}$-terminal mediates the activation of internal signal transduction. Extracellular (ECL) and intracellular (ICL) domains are important for the interaction with nonapeptides and G-proteins respectively, while transmembrane domains (TMs) are connected with both functions (Zingg and Laporte, 2003; Koehbach et al., 2013).

AVPR1a, AVPR1b and OXTR play important roles in a range of social behaviors. AVPR1b is produced in the anterior pituitary gland, where it mediates the secretion of the adrenocorticotropic hormone (ACTH) (Koshimizu et al., 2012). ACTH is an important component of the hypothalamic-pituitary-adrenal (HPA) axis, a key system controlling a range of important adaptive behaviors, including circadian rhythms, the stress reaction and responses to fear (Kalsbeek et al., 2012; De Oliveira et al., 2013; Terenina et al., 2013). Through a connection with ACTH, HPA and other neuronal mechanisms, mutations in AVPRIb have been associated with altered responses to stress and aggressive behavior in Sus scrofa (Muráni et al., 2010; Terenina et al., 2013; Lucion and Bortolini, 2014). AVPR1b knockout mice showed normal predatory behavior, while presenting a reduced level of aggression against individuals of their own species (Wersinger et al., 2007). Treatment with AVPR1a antagonists has been shown to result in reduced anxiety and offensive aggression in rats and hamsters, respectively (Liebsch et al., 1996; Keverne and Curley, 2004; Ferris et al., 2006). In addition, variation in the promoter region of AVPRla could explain differences in behavior within and between species, including humans, bonobos, and chimpanzees (Staes et al., 2016). OXTR is associated with a wide range of phenotypes. It is expressed in the uterus, where it controls uterine contraction, and in the mammary glands, where it regulates milk ejection; in the brain, it governs social learning and emotion (Kimura et al., 1992; Guzmán et al., 2013a,b; Yamashita and Kitano,
2013). Finally, AVPR2 stimulates urine concentration and helps maintain water homeostasis (Carter, 2013; Koshimizu et al., 2012; Yamashita and Kitano, 2013). It is the only AVP receptor that is not directly involved with behavior; however, it increases the expression of AVPR1a through the formation of heterodimers with that receptor (Terrillon et al., 2004). In a wider context, the increased availability of resources resulting from the contact with humans may have impacted on the diet of domesticated animals, potentially leading to metabolic changes, which may be reflected in the OXT-AVP system.

Recently, Paré et al. (2016) explored the evolutionary forces acting on the four receptors OXTR, AVPR1a, $A V P R 1 b$, and $A V P R 2$ in a set of 35 placental mammal orthologs. They found evidence for a dynamic scenario, in which $O X T R$ was under evolutionary constraint, while AVPR1a, AVPR1b, and AVPR2 exhibited elevated rates of evolution, indicating a relaxation of selective pressures, or even positive selection of novel vasopressin receptor variants in the Placentalia (Paré et al., 2016). In the light of these recent findings of the genetic variability of the OXTAVP system, we aimed to explore whether amino acid changes at $A V P, O X T, O X T R, A V P R 2, A V P R 1 a$, and $A V P R 1 b$ could be associated with the animal domestication process.

\section{Material and Methods}

Studies of domestication are commonly based on a comparison between domesticated and their putative wild ancestral lineages. Assuming that wild and domesticated species carry different alleles, it is possible to identify the ancestral allele as the most common one in the studied mammals and to thus reconstruct the genetic background on which domestication occurred. This is relatively straightforward where the wild ancestral species is known, e.g. in the case of the dog, which we compared to the gray wolf (Canis lupus; PRJNA266585; Li et al., 2013). However, extant wild species have followed their own evolutionary path from the moment of divergence to the present time (Freedman et al., 2014). It is therefore ideal to use the ancient genomes of the ancestral species for comparison wherever possible. Ancestral DNA (aDNA) sequences were available for the putative wild ancestors of cattle (auroch, Bos primigenius; PRJNA294709; Park et al., 2015) and horse (two Late Pleistocene equines; PRJEB7537; Schubert et al., 2014). Finally, a further challenge lies in the fact that the wild ancestral species of many domesticated animals are either extinct or unknown. Wherever the putative ancestral species were unavailable, we therefore compared the domesticated species with their phylogenetically closest wild relative.

While the literature offers numerous definitions of a domesticated species (Larson and Burger, 2013), here we refer to a domesticated animal as "one whose mate choice is influenced by humans and whose tameness and tolerance of 
humans are genetically determined" (Driscoll et al., 2009). Based on morphological and behavioral phenotypes shared between humans and a handful of domesticated species, Homo sapiens can be referred to as a self-domesticated species (Hare, 2017). Therefore, to avoid methodological bias, we ran evolutionary tests for gene sets with and without data from Homo sapiens and its phylogenetically closest (wild) relative, Pan troglodytes.

\section{Data mining}

The coding sequences of $A V P, O X T$ (including neurophysin and signal peptide sequences), OXTR, AVPR2, $A V P R 1 a$, and $A V P R 1 b$ of 19 domesticated and 21 wild placental mammals (Table S1) were obtained from the NCBI GenBank genomic database (http://www.ncbi.nlm.nih.gov) and Ensembl (http://www.ensembl.org). Sequences with an identity value $\geq 98 \%$, a minimum score of 200 and a statistically significant E-value (i.e. Sus scrofa, Bos primigenius, Felis silvestris, Capra aegagrus, Ovis orientalis, Canis lupus and ancient horse) were aligned using the MUSCLE algorithm (Edgar, 2004) implemented in Mega 6.0 (Tamura et al., 2011). In order to maximize the number of speciesspecific sequences per gene, the set of homologous sequences analyzed for each gene varied, ranging from 26 for $A V P$ and $O X T R$, to 36 for AVPRIb (Supplementary material - Alignment Analyses 1-6).

\section{Data analysis}

To detect positive selection, we applied the NsSites test, implemented in PAML 4.9. This approach is based on an interspecific phylogenetic comparison of nonsynonymous and synonymous substitution rates in protein-coding genes. The type of selection is indicated by the value of the nonsynonymous/synonymous rate ratio $\omega=\mathrm{dN} / \mathrm{dS}$. We compared neutral models with alternative models allowing positive selection, performing a pairwise comparison of two likelihood ratio tests (LRTs) of the following PAML models: M1a vs. M2a and M7 vs. M8 (Yang, 2007). Due to the inclusion of ancient genome sequences, such as that of the aurochs (Bos primigenius; Park et al., 2015) and two Late Pleistocene equines (CGG10022 and CGG10023; Schubert et al., 2014), all sites in the alignment were used in the analysis ("clean data $=0$ ").

The Bayes Empirical Bayes (BEB) approach implemented in CODEML was used to select sites potentially under positive selection. Phylogenetic trees constructed in accordance with the published phylogenies of primates (Perelman et al., 2011) and mammals (Meredith et al., 2011; Song et al., 2012) were used as input for PAML 4.9. For sites with a high (>90\%) posterior probability of being under positive selection in BEB, we determined the Grantham score (GS), a chemical measure for the fit between an amino acid and the one replacing it in a missense substitution. Based on their GS, amino acid changes were categorized as conservative (GS $0-50$ ), moderately conservative
(51-100), moderately radical (101-150), and radical (> 151) (Grantham, 1974; Li et al., 1984).

Importantly, while the NsSites test has been developed to detect natural selection, we assume that signals of positive selection in domesticated animals reflect artificial selection. Furthermore, we applied the posterior Clade D test, a branch-site model implemented in PAML, to compare the evolutionary rates found in a domesticated species either to its putative wild ancestor species or to the most closely-related wild species whose genome is available. The phylogeny was divided into two clades, and an LRT was used to evaluate divergences in selective pressure between them, as indicated by different $\omega$ ratios. This model assumes two classes of sites, foreground and background sites, which were compared to the neutral model M1a using an LRT with three degrees of freedom. Importantly, the phylogenetic tree was built in a mirrored way, comparing the domesticated animals on the foreground branches with the wild animals on the background branches, always using the same number of species per branch. Any divergence in the evolutionary rates ( $\omega$ ratios) of corresponding domesticated and wild branches can be thought to be due to either positive selection or the relaxation of natural selection. Figures S1-S6 show two structurally different trees for each analyzed protein: the tree at the top is a "mirrored tree", which artificially separates the foreground branches (domesticated animals) from the background branches (wild animals; e.g., Figure S1.1). The tree at the bottom represents the actual phylogenetic tree, as published in the literature (e.g., Figure S1.2). Importantly, the actual phylogenetic trees were used in the NsSites analyses, while the mirrored trees were used in the branch comparisons for Clade D and RELAX (see below).

We searched for signals of relaxed selection using the online tool RELAX (http://test.datamonkey.org/relax; Delport et al., 2010; Wertheim et al., 2015). RELAX allows to infer the variation of evolutionary rates both between sites and across branches. The relaxation of natural selection is indicated by an increase (for $\omega<1$ ) or decrease (for $\omega>1$ ) of $\omega$ toward 1 . In the context of models that compared two or more branches in a phylogeny (branch-site models), different proportions of sites fall into each $\omega$ category, leading to two different effects: On the one hand, the values inferred for the each selection category can move toward 1 . On the other hand, the proportions of sites belonging to the different categories can change in such a way that more sites are assigned to categories with $\omega$ values closer to 1 (Wertheim et al., 2015). It should be stressed that RELAX uses the $K$-value, which measures the selection strength of each branch. A $K>1$ indicates that the respective branch has been subjected to stronger purifying selection in relation to the other branch(es). Alternatively, sites that are under moderate positive selection in the reference branch are subjected to stronger positive selection. $K$-values $<1$ for a 
branch indicate the relaxation of purifying selection (Wertheim et al., 2015).

\section{Results and Discussion}

Domesticated species are subject to severe artificial selection. They can therefore be expected to present genetic changes when compared with their putative wild ancestral species, or with phylogenetically related species in the wild. Here, we used classical approaches to infer positive selection through the interspecific phylogenetic comparison of six candidate genes and the evaluation of different evolutionary rates between wild and domesticated species. In the present context we assume that a signal of positive selection can reflect artificial selection.

\section{Analysis of positive selection}

Initially, we searched for relevant selection signals to answer our original question whether changes in $A V P$, OXT, OXTR, AVPR2, AVPR $1 a$, and AVPR $1 b$ could be associated with the animal domestication process. Therefore, we only considered sites with significant differences in the amino acids between a domesticated species and its puta- tive wild ancestor or a phylogenetically closely related wild species.

The NSsites analysis of $A V P R 1 b$ and $A V P R 2$ revealed that the different selective pressures acting in wild and captive environments left molecular footprints at specific sites within these genes in both sets of animals (Table 1).

Specifically, AVPR $1 b$ was found to harbor one site with a posterior probability $>95 \%$ of being under positive selection (position 404 of AVPR1b; $\omega=1.429, p=0.001$ ). AVPR2 $(\omega=1.49, p=0.001)$ was found to contain three such sites (positions 3, 257 and 302 of AVPR2; Table S2; Figure 1; see also Material and Methods). The evolutionary model that best fitted both $A V P R 1 b$ and $A V P R 2$ was M8 ( $p$ $=0.001$ and $p<0.001$, respectively; Table S2).

AVPR1b position 404 is located within the C-terminal, an important region for G-protein interaction (Zingg and Laporte, 2003). Although this region is highly variable across mammals, all carnivores carry the same amino acid, suggesting high evolutionary conservation within this clade (Table S3).

It is worthy of note that the Alpaca (Vicugna pacos) also differs from its closest wild relative at position 404 of AVPR1b, with a moderately radical change $(\mathrm{GS}=121)$.

Table 1 - Sites within $A V P R 1 B$ and $A V P R 2$ with a high probability ( $>90 \%)$ of being under positive selection in domesticated animals $(\omega$ value $>1$ for Model 8).

\begin{tabular}{|c|c|c|c|c|c|c|}
\hline \multicolumn{7}{|c|}{ AVPR1B } \\
\hline Domain & AA position & Wild species ${ }^{1}$ & AA & Domesticated species & GS & Probability $^{2}$ \\
\hline \multirow[t]{4}{*}{ C-TERMINAL } & 404 & Camelus bactrianus & Val-Glu & Vicugna pacos & 121 & 0.964 \\
\hline & 404 & Octodon degus & Val-Ala & Cavia porcellus & 64 & 0.964 \\
\hline & 404 & Marmota marmota & Ala-Glu & Chinchilla lanigera & 107 & 0.964 \\
\hline & 404 & Ochotona princeps & Gly-Gln & Oryctolagus cuniculus & 98 & 0.964 \\
\hline \multicolumn{7}{|c|}{ AVPR2 } \\
\hline \multirow[t]{7}{*}{ N-TERMINAL } & 3 & Marmota marmota & Leu-Ser & Cavia porcellus & 145 & 0.994 \\
\hline & 3 & Camelus ferus & Leu-Met & Camelus dromedarius & 15 & 0.994 \\
\hline & 3 & Sus scrofa & Arg-Ala & Sus scrofa familiaris & 112 & 0.994 \\
\hline & 3 & Bos primigenius & Ser-Met & Bos taurus & 135 & 0.994 \\
\hline & 3 & Ovis orientalis & Cys-Met & Ovis aries & 196 & 0.994 \\
\hline & 3 & Capra aegagrus & Ser-Met & Capra hircus & 135 & 0.994 \\
\hline & 3 & Ailuropoda melanoleuca & Met-Thr & Mustela putorius furo & 81 & 0.994 \\
\hline \multirow[t]{3}{*}{ ICL3 } & 257 & Peromyscus maniculatus & Ile-Ser & Mus musculus & 142 & 0.922 \\
\hline & 257 & Ictiodomys tridecemlineatus & Arg-Thr & Mesocricetus auratus & 71 & 0.922 \\
\hline & 257 & Ochotona princeps & His-Ser & Oryctolagus cuniculus & 89 & 0.922 \\
\hline \multirow[t]{6}{*}{ ECL3 } & 302 & Marmota marmota & Leu-Arg & Cavia porcellus & 102 & 0.911 \\
\hline & 302 & Ancient Horse & Pro-Arg & Equus caballus & 103 & 0.911 \\
\hline & 302 & Bos primigenius & Pro-Arg & Bos taurus & 103 & 0.911 \\
\hline & 302 & Capra aegagrus & His-Arg & Capra hircus & 29 & 0.911 \\
\hline & 302 & Felis silvestris & Pro-Arg & Felis catus & 103 & 0.911 \\
\hline & 302 & Ailuropoda melanoleuca & Leu-Val & Mustela putorius furo & 32 & 0.911 \\
\hline
\end{tabular}

${ }^{1}$ Putative ancestral species or phylogenetically related wild species; ${ }^{2}$ probability of being under positive selection in the Bayes Empirical Bayes (BEB) analysis. 

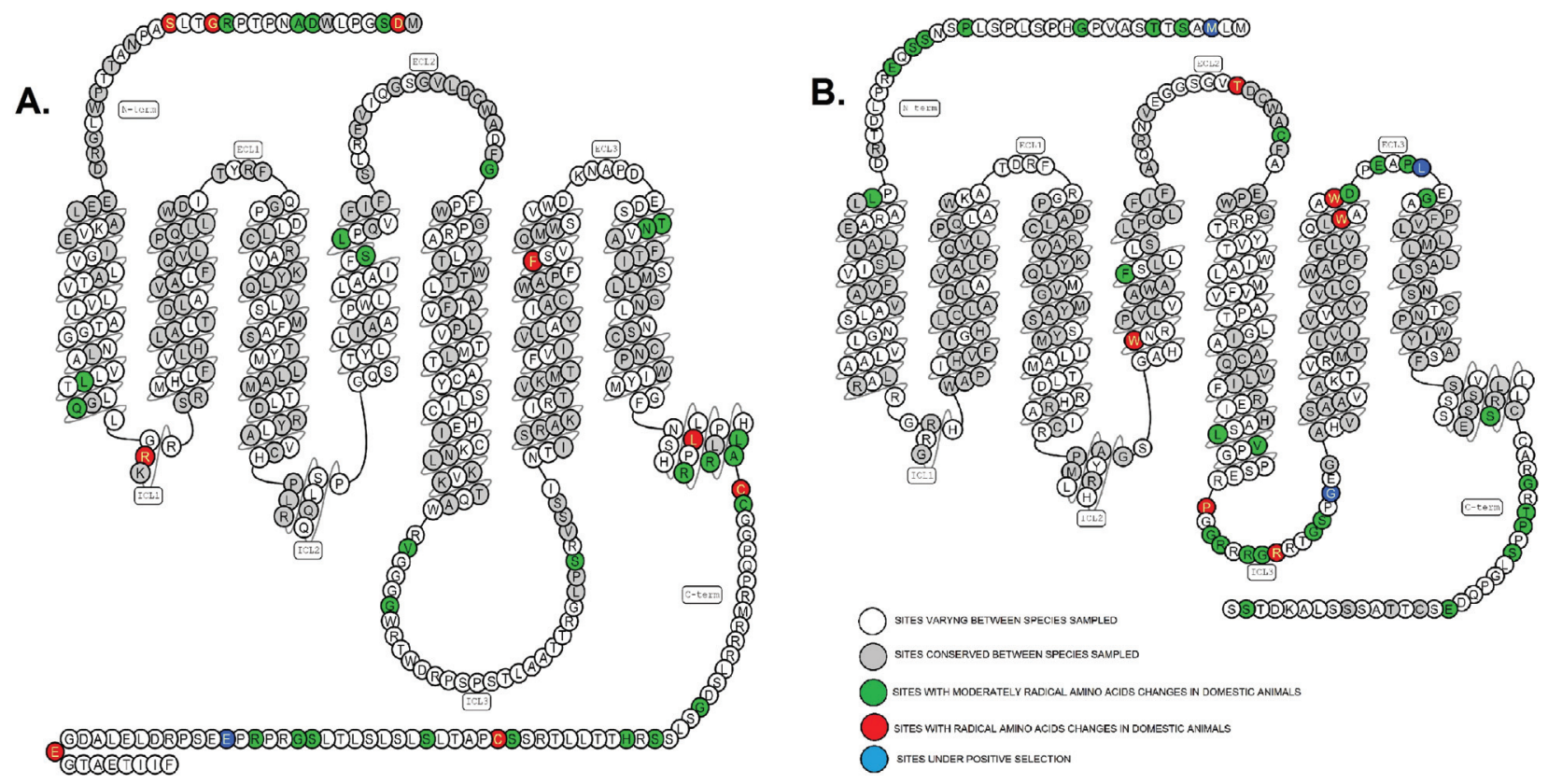

Figure 1 - Snake plot of (A) AVPR1b and (B) AVPR2 (Reference: www.gpcrdb.org).

Since AVPR1b is known to play an important role in behavior, this nonsynonymous substitution could have been important for the domestication of Alpaca in Pre-Hispanic South America (Westbury et al., 2016). A role for AVPR1b in the behavioral changes associated with domestication is consistent with findings of a reduced basal plasma levels of $\mathrm{ACTH}$ in foxes subjected to rigorous artificial selection for domestication phenotypes over more than 50 years (Trut et al., 2009): ACTH is an important hormone of the hypothalamic-pituitary-adrenal (HPA) axis, and its secretion in this axis is modulated by the AVPR 1 b receptor (Kalsbeek et al., 2012; Terenina et al., 2013). Interestingly, Chinchilla lanigera also carries a moderately radical change at this site. However, the chinchilla was domesticated for its fur (Baranowski et al., 2013), and the impact of the domestication process on its behavior is still uncertain.

Within AVPR2, positions 3, 257 and 302 were found to be under positive selection (Tables 1 and S2). Located in the N-terminal region of the protein, position 3 has a $99 \%$ probability of being under positive selection. Seven domesticated species differed from their wild relatives at this position: Cavia porcellus, Camelus dromedarius, Sus scrofa familiaris, Bos taurus, Ovis aries, Capra hircus and Mustela putorius furo. Five of these seven modifications led to an at least moderately radical amino acid change. Interestingly, four domesticated Artiodactyla species carry a methionine at this position (Camelus dromedarius, Bos taurus, Ovis aries, and Capra hircus) (Tables 1 and S3). Based on its distribution across the species, Met can be considered the ancestral amino acid. It should be stressed that this amino acid is maintained only in domesticated Artiodactyla, while three other amino acids, Leu, Ser and Cys, are found in their respective wild relatives. Assuming that
Met is not the ancestral amino acid, this can be suggested as an instigating case of molecular convergence in domesticated animals (Zhang and Kumar, 1997; Melville et al., 2006). Therefore, it is possible that this site might have evolved at a different rate in both sets of animals, at least within the Artiodactyla clade.

Five domesticated animals (Equus caballus, Bos taurus, Cavia porcellus, Capra hircus, and Felis catus) had an arginine at position 302, which is under positive selection with a probability of $>90 \%$ (ECL3, Figure 1; Tables 1, S2 and S3). Like the Met at position 3, this is another case where an ancestral amino acid seems has been retained in the domesticated branch. However, the presence of the same amino acid concomitantly in the different species due to convergence cannot be discarded (Table S3). Finally, position 257 (ICL2, Figure 1) has a probability of $92 \%$ of being under positive selection. At this site we found three domestic Glires (rodents and lagomorphs) presenting three different amino acid changes (Tables 1 and S3) compared to their wild relatives.

Changes in AVPR2 are associated with metabolic functions, such as homeostasis, rather than behavior (Carter, 2013; Koshimizu et al., 2012; Yamashita and Kitano, 2013). However, genomic analyses have revealed that genes involved in metabolism are under selection in domesticated animals, including cats (Felis catus) (Montague et al., 2014). Our results support the idea that dietary manipulation and an increased availability of resources provided by humans, including abundant water supply, may have led to functional changes in AVPR2 in domesticated animals.

Our analyses have shown that $O X T, A V P$ and AVPRla are under purifying selection. As expected, OXTR 
seems to be under evolutionary constraint in the set of mammals considered here, corroborating earlier studies (Paré et al., 2016). However, despite this general pattern, there is evidence that $O X T R$ has been under positive selection in some New World monkey branches, where it has coevolved with taxon-specific OXT forms (Vargas-Pinilla et al., 2015). Of note, the inclusion of Homo sapiens and our wild relative Pan troglodytes yielded the same results (data not shown).

\section{Relaxation of selective constraints}

A relaxation of selective pressure has previously been described for some domesticated species, including dogs and horses (Price, 1999; Zeder, 2012; Li et al., 2013; Schubert et al., 2014). Analysis of the Clade D model showed that evolutionary rates differed between the branches carrying domesticated animals and those carrying their respective wild relatives for all genes in the present study. However, $\omega$ values were below 1 (Table S4), indicating neutrality or relaxed selective constraint. We therefore applied RELAX, a program specialized in the identification of relaxed constraint, on the same dataset. Results from these analyses showed a relaxation of selective constraints on AVPRla in the domesticated branch $(\mathrm{k}=0.47, p=$ $0.003)$. Since within-group aggression can imply the loss of one or several animals, the control of social behavior is extremely important in the domestication process. Within AVPR1a, the regions with the highest level of variation were the N-terminal and ECL3 domains, which interact with the AVP and OXT nonapeptides (Zingg and Laporte, 2003; Koehbach et al., 2013). AVPR1a is known for its role in behavior, particularly the control of aggression and anxiety (Staes et al., 2016). Nevertheless, the administration of AVPR1a antagonists has been found to reduce anxiety in rats (Liebsch et al., 1996) and offensive aggression in hamsters (Ferris et al., 2006). Thus, a relaxation of selective constraint on the AVPRla gene in domesticated animals may reflect the fact that aggressive and anxiolytic behaviors play a lesser part in fitness and survival in captivity than in the wild.

$A V P(\mathrm{k}=2.5, p<0.001)$ and $O X T(\mathrm{k}=1.4, p=0.04)$ were found to be under moderate purifying selection in the reference branches (wild animals), as previously suggested by our group (Paré et al., 2016). In the branch test, purifying selection was found to be even stronger in domesticated animals. In other words, it seems that there is a higher constraint to change in domesticated animals than in wild ones. Notwithstanding, AVP-coded neurophysin (NP) has a relatively high level of variation, with some amino acid changes in rodents, carnivores and artiodactyls. For example, radical changes with respect to the wild species are observed at position NP-42 in Canis lupus, Sus scrofa, and Capra aegagrus (GS=159-192), with an ancestral cysteine present in the ancient horse and the remaining 23 animals. In $O X T$, the most variable region is also that encoding NP. Radical modifications in wolf (Asp35Leu), mouflon sheep
(Cys41Gly) and wild goat (Cys41Tyr) were observed compared to their putative domesticated correspondents. The same pattern of change was found in the wild goat (Cys52Leu and Cys116Phe), wild boar (Cys116Arg) and mouflon sheep (Gly116Cys; Table S3; all artiodactyls).

Additionally, despite the conservation of AVP across placental mammals, we identified a lysine (Lys) at position 8 , both in the domestic pig (Sus scrofa familiaris) and the wild boar (Sus scrofa). The preservation of this rare change in Sus scrofa familiaris, even after severe artificial selection, may indicate an indispensable functional importance of AVP-8Lys in the $S u$ s genus. The other receptor genes, $A V P R 1 b(\mathrm{k}=1.04, p=0.6), A V P R 2(\mathrm{k}=0.99, p=0.9)$ and $\operatorname{OXTR}(\mathrm{k}=1.1, p=0.1)$, did not show signals of a relaxation of natural selection. This is expected since we identified positive selection at least in the $A V P R 1 b$ and $A V P R 2$ genes, demonstrating the contrasting selection pressures in wild and captive environments.

Tameness and the reduction of stress, fear and anxiety are traits associated with domestication (Künzl and Sachser, 2009). Our results indicate that the $O X T$ and $A V P$ systems likely play an important role in this process. Overall, the sites underlying the evolutionary signals found here could be involved in aggressive and stress control phenotypes (e.g. C-terminal changes at position 404 of AVPR1b), but also in physiological traits related to conditions caused by humans (e.g. AVPR2-3Met in domesticated Artiodactyla). Importantly, genes underlying behavior that would be selected for in the natural environment, such as aggression, would be subject to a relaxation of selective pressures in the captive environment, as suggested by our results for AVPR1a. Last but not least, the analyses of selective constraint that included humans and chimpanzees did not differ from those excluding them.

\section{Conclusion}

In nature, taxon-specific genetic changes have been associated with taxon-specific phenotypes, including adaptive behaviors. Following this idea, we describe amino acid changes at two GPCR receptors (AVPR1b and AVPR2) that are under positive selection comparing domesticated and wild species. While $A V P$ and $O X T$ were identified as under strong purifying selection, AVPRla shows a clear signal of relaxed selective pressure in domesticated animals. Based on the known function of these genes, these patterns of selection may be thought to be associated with physiological and behavior modifications needed for domesticated animals to coexist and coevolve with humans in their constructed niches.

\section{Acknowledgments}

This research was financially supported by the Coordenação de Aperfeiçoamento de Pessoal de Nível Superior (CAPES) and the Conselho Nacional de Desenvolvimento 
Científico e Tecnológico (CNPq; Programa de Apoio a Núcleos de Excelência-PRONEX).

\section{References}

Albert FW, Somel M, Carneiro M, Aximu-Petri A, Halbwax M, Thalmann O, Blanco-Aguiar JÁ, Plyusnina IZ, Trut L, Villafuertes R, et al. (2012) A comparison of brain gene expression levels in domesticated and wild animals. PLoS Genet 8:1-16.

Baranowski P, Wróblewska M, Nowak P and Pezinska K (2013) Biometry of the skull of wild and farm long-tailed chinchilla (Chinchila laniger, Molina, 1782). Int $\mathrm{J}$ Morphol 31:1003-1011.

Cagliani R, Fumagalli M, Pozzoli U, Riva S, Cereda M, Comi GP, Pattini L, Bresolin N and Sironi M (2009) A complex selection signature at the human AVPR1B gene. BMC Evol Biol 9:123.

Carter CS (2013) Oxytocin pathways and the evolution of human behavior. Annu Rev Psychol 65:17-39.

Darwin C (1868) The Variation of Animals and Plants Under Domestication, Vols. I and II. John Murray, London.

De Oliveira AR, Reimer AE, Reis FM and Brandão ML (2013) Conditioned fear response is modulated by a combined action of the hypothalamic-pituitary-adrenal axis and dopamine activity in the basolateral amygdala. Eur Neuropsychopharmacol 23:379-389.

Delport W, Poon AF, Frost SD and Kosakovsky Pond SL (2010) Datamonkey 2010: A suite of phylogenetic analysis tools for evolutionary biology. Bioinformatics 26:2455-2457.

Driscoll CA, Macdonald DW and O'Brien SJ (2009) From wild animals to domestic pets, an evolutionary view of domestication. Proc Natl Acad Sci U S A 106:9971-9978.

Edgar RC (2004) MUSCLE: Multiple sequence alignment with high accuracy and high throughput. Nucleic Acids Res 32:1792-1797.

Ferris CF, Lu SF, Messenger T, Guillon CD, Heindel N, Miller M, Koppel G, Robert Bruns F and Simon NG (2006) Orally active vasopressin V1a receptor antagonist, SRX251, selectively blocks aggressive behavior. Pharmacol Biochem Behav 83:169-174.

Freedman AH, Gronau I, Schweizer RM, Ortega-Del Vecchyo D, Han E, Silva PM, Galaverni M, Fan Z, Marx P, LorenteGaldos B, et al. (2014) Genome sequencing highlights the dynamic early history of dogs. PLOS Genetics 10:e1004016.

Gimpl G and Fahrenholz F (2001) The oxytocin receptor system: structure, function, and regulation. Physiol Rev 81:629-683.

Grantham R (1974) Amino acid difference formula to help explain protein evolution. Science 185:862-864.

Guzmán YF, Tronson NC, Jovasevic V, Sato K, Guedea AL, Mizukami H, Nishimori K and Radulovic J (2013a) Fearenhancing effects of septal oxytocin receptors. Nat Neurosci 16:1185-1187.

Guzmán YF, Tronson NC, Sato K, Mesic I, Guedea AL, Nishimori K and Radulovic J (2013b) Role of oxytocin receptors in modulation of fear by social memory. Psychopharmacology (Berl) 231:2097-2105.

Hare B (2017) Survival of the friendliest: Homo sapiens evolved via selection for prosociality. Annu Rev Pyschol 68:155186.
Kalsbeek A, van der Spek R, Lei J, Endert E, Buijs RM and Fliers E (2012) Circadian rhythms in the hypothalamo-pituitaryadrenal (HPA) axis. Mol Cell Endocrinol 349:20-29.

Keverne EB and Curley JP (2004) Vasopressin, oxytocin and social behavior. Neurobiol Behav 14:777-783.

Kimura T, Tanizawa O, Mori K, Brownstein MJ and Okayama $\mathrm{H}$ (1992) Structure and expression of a human oxytocin receptor. Nature 356:526-529.

Koehbach J, Stockner T, Bergmayr C, Muttenthaler M and Gruber CW (2013) Insights into the molecular evolution of oxytocin receptor ligand binding. Biochem Soc Trans 41:197-204.

Koshimizu TA, Nakamura K, Egashira N, Hiroyama M, Nonoguchi $\mathrm{H}$ and Tanoue A (2012). Vasopressin V1a and V1b receptors: From molecules to physiological systems. Physiol Rev 92:1813-1864.

Künzl C and Sachser N (1999) The behavioral endocrinology of domestication: A comparison between the domestic guinea pig (Cavia aperea $f$. porcellus) and its wild ancestor, the cavy (Cavia aperea). Horm Behav 35:28-37.

Larson G and Burger J (2013) A population genetics view of animal domestication. Trends Genet 29:197-205.

Liebsch G, Wotjak CT, Landgraf R and Engelmann M (1996) Septal vasopressin modulates anxiety-related behaviour in rats. Neurosci Lett 217:101-104.

Li WH, Wu CI and Luo CC (1984) Non randomness of point mutation as reflected in nucleotide substitutions in pseudogenes and its evolutionary implications. J Mol Evol 21:58-71.

Li Y, von Holdt BM, Reynolds A, Boyko AR, Wayne RK, Wu DD and Zhang YP (2013) Artificial selection on brain-expressed genes during the domestication of dog. Mol Biol Evol 30:1867-1876.

Lucion AB and Bortolini MC (2014) Mother-pup interactions: Rodents and humans. Front Endocrinol 5:17.

Marsden CD, Ortega-Del Vecchyo D, O'Brien DP, Taylor JF, Ramirez O, Vilaf C, Marques-Bonete T, Schnabdel RD, Waynea RK and Lohmueller KE (2016) Bottlenecks and selective sweeps during domestication have increased deleterious genetic variation in dogs. Proc Natl Acad Sci U S A 113:152-157.

McCall C and Singer T (2012) The animal and human neuroendocrinology of social cognition, motivation and behavior. Nat Neurosci 15:681-688.

Melville J, Harmon LJ and Losos JB (2006) Intercontinental community convergence of ecology and morphology in desert lizards. Proc Biol Sci 273:557-563.

Meredith RW, Janecka JE, Gatesy J, Ryder OA, Fisher CA, Teeling EC, Goodbla A, Eizirik E, Simão TLL, Stadler T, et al. (2011) Impacts of the Cretaceous terrestrial revolution and $\mathrm{KPg}$ extinction on mammal diversification. Science 334:521-524.

Montague MJ, Li G, Gandolfi B, Khan R, Aken BL, Searle SMJ, Minx P, Hillier LW, Kobolt DC, Davis BW, et al. (2014) Comparative analysis of the domestic cat genome reveals genetic signatures underlying feline biology and domestication. Proc Natl Acad Sci U S A 111:17230-17235.

Muráni E, Ponsuksili S, D’Eath RB, Turner SP, Kurt E, Evans G, Thölking L, Klont R, Foury A, Mormède P, et al. (2010) Association of HPA axis-related genetic variation with stress reactivity and aggressive behavior in pigs. BMC Genetics 11:74.

Paré P, Paixão-Côrtes VR, Tovo-Rodrigues L, Vargas-Pinilla P, Viscardi LH, Salzano FM, Henkes LE and Bortolini MC (2016) Oxytocin and arginine vasopressin receptor evolu- 
tion: implications for adaptive novelties in placental mammals. Genet Mol Biol 39:646-657.

Park SD, Magee DA, McGettigan PA, Teasdale MD, Edwards CJ, Lohan AJ, Murphy A, Braud M, Donoghue MT, Liu Y, et al. (2015) Genome sequencing of the extinct Eurasian wild aurochs, Bos primigenius, illuminates the phylogeography and evolution of cattle. Genome Biol 16:234.

Perelman P, Johnson WE, Roos C, Seuánez HN, Horvath JE, Moreira MAM, Kessing B, Pontius J, Roelke M, Rumpler Y, et al. (2011) A molecular phylogeny of living primates. PLoS Genetics 7:1-17.

Price EO (1999) Behavioral development in animals undergoing domestication. Appl Anim Behav Sci 65:245-271.

Schubert M, Jónsson H, Chang D, Schubert M, Jónsson H, Chang D, Der Sarkissian C, Ermini L, Ginolhac A, Albrechtsen A, et al. (2014) Prehistoric genomes reveal the genetic foundation and cost of horse domestication. Proc Natl Acad Sci U S A 111:E5661-E5669.

Slusarz MJ, Sikorska E and Slusarz R (2013) Interactions of vasopressin and oxytocin receptors with vasopressin analogues substituted in position 2 with 3,3'-diphenylalanine - a molecular docking study. J Pept Sci 19:118-126.

Song S, Liu L, Edwards SV and Wu S (2012) Resolving conflict in eutherian mammal phylogeny using phylogenomics and the multispecies coalescent model. Proc Natl Acad Sci U S A 109:14942-14947.

Staes N, Weiss A, Helsen P, Korody M, Eens M and Stevens JM (2016) Bonobo personality traits are heritable and associated with vasopressin receptor gene 1a variation. Sci Rep 6:38193.

Tamura K, Perterson D, Peterson N, Stecher G, Nei M and Kumar S (2011) MEGA5: Molecular Evolutionary Genetics Analysis using maximum likelihood, evolutionary distance, and maximum parsimony methods. Mol Biol Evol 28:27312739.

Terenina E, Babigumira BM, Le Mignon GL, Bazovkina D, Rousseau S, Salin F, Bendixen C and Mormede P (2013) Association study of molecular polymorphisms in candidate genes related to stress responses with production and meat quality traits in pigs. Domest Anim Endocrinol 44:81-97.

Terrillon S, Barberis C and Bouvier M (2004) Heterodimerization of V1a and V2 vasopressin receptors determines the interaction with arrestin and their trafficking patterns. Proc Natl Acad Sci U S A 101:1548-1553.

Trut L, Oskina I and Kharlamova A (2009) Animal evolution during domestication: the domesticated fox as a model. Bioessays 31:349-360.

Vargas-Pinilla P, Paixão-Côrtes VR, Paré P, Tovo-Rodrigues L, Vieira CMAG, Xavier A, Comas D, Pissinati A, Sinigaglia M, Rigo MM, et al. (2015) Evolutionary pattern in the OXT-OXTR system in primates: Coevolution and positive selection footprints. Proc Natl Acad Sci U S A 112:88-93.

Wersinger SR, Caldwell H, Christiansen M and Young W (2007) Disruption of the vasopressin $1 \mathrm{~b}$ receptor gene impairs the attack component of aggressive behavior in mice. Genes Brain Behav 6:653-660.

Wertheim JO, Murrell B, Smith MD, Kosakovsky Pond SL and Scheffler K (2015) RELAX: Detecting relaxed selection in a phylogenetic framework. Mol Biol Evol 32:820-832.

Westbury M, Prost S, Seelenfreund A, Ramírez J-M, MatisooSmith EA and Knapp M (2016) First complete mitochondrial genome data from ancient South American camelids the mystery of the chilihueques from Isla Mocha (Chile). Sci Rep 6:38708.
Wiener P and Wilkinson S (2011) Deciphering the genetic basis of animal domestication. Proc R Soc B-Biol Sci 278:31613170 .

Wright D (2015) The genetic architecture of domestication in animals. Bioinform Biol Insights 9:11-20.

Yamashita K and Kitano T (2013) Molecular evolution of the oxytocin-oxytocin receptor system in eutherians. Mol Phylogenet Evol 67:520-528.

Yang Z (2007) PAML 4: A program package for phylogenetic analysis by maximum likelihood. Comput Appl Biosci 24:1586-1591.

Young LJ and Flanagan-Cato LM (2012) Oxytocin, vasopressin and social behavior. Horm Behav 61:227-229.

Zhang J and Kumar S (1997) Detection of convergent and parallel evolution at the amino acid sequences level. Mol Biol Evol 14:527-536

Zeder MA (2012) Pathways to animal domestication. In: Gepts P, Famula TR, Bettinger RL, Brush SB, Damania AB, McGuire PE and Qualset CO (eds) Biodiversity in Agriculture: Domestication, Evolution and Sustainability. Cambridge Univ. Press, Cambridge, pp 227-259.

Zingg HH and Laporte SA (2003) The oxytocin receptor. Trends Endocrinol Metab 14:222-227.

\section{Supplementary material}

The following online material is available for this article: Alignment analyses - Alignments (1-6) used in the analyses of the different species of wild and domesticated mammals. Table S1 - Species of wild and domestic placental mammals available in genome data banks and used in the analyses.

Table S2 - Estimated parameters under different codon substitution models for $O X T$ and $A V P$ system genes.

Table S3 - OXT-AVP system amino acid positions with moderately radical or radical changes according to the Grantham Score (GS > 101).

Table S4 - Results of the Clade D analysis.

Figure S1 - Phylogenetic trees of 26 species of mammals used in $A V P$ analysis, 1: mirrored tree, 2: phylogenetic tree.

Figure S2 - Phylogenetic trees of 26 species of mammals used in AVPR Ia analysis, 1: mirrored tree, 2: phylogenetic tree.

Figure S3 - Phylogenetic trees of 26 species of mammals used in AVPR 1b analysis, 1: mirrored tree, 2: phylogenetic tree.

Figure S4 - Phylogenetic trees of 26 species of mammals used in AVPR2 analysis, 1: mirrored tree, 2: phylogenetic tree.

Figure S5 - Phylogenetic trees of 26 species of mammals used in $O X T$ analysis, 1: mirrored tree, 2: phylogenetic tree. Figure S6 - Phylogenetic trees of 26 species of mammals used in $O X T R$ analysis, 1: mirrored tree, 2: phylogenetic tree.

Associate Editor: Loreta B. Freitas

License information: This is an open-access article distributed under the terms of the Creative Commons Attribution License (type CC-BY), which permits unrestricted use, distribution and reproduction in any medium, provided the original article is properly cited. 